\title{
Riscos no trabalho em olarias e seu entendimento por parte dos trabalhadores
}

\author{
Riesgos en el trabajo en alfarerías y su entendimiento por parte de los \\ trabajadores
}

Work risks in potteries and its understanding on the part of the workers

\author{
José Paulo Braccini Fagundes ${ }^{1}$
}

\begin{abstract}
Resumo
Por se tratar muitas vezes de um labor em condições precárias e com recursos humanos com níveis de escolaridade geralmente baixos, o trabalho desenvolvido em olarias merece atenção no que tange à adequada informação dos trabalhadores sobre os riscos existentes nos ambientes de trabalho. Em alguns casos, observamse condições extremamente precárias com relação à segurança e saúde no trabalho, sem a devida proteção das máquinas e dos trabalhadores ou instalações elétricas inadequadas. O mapa de riscos, representação gráfica dos fatores capazes de acarretar prejuízos à saúde do trabalhador, foi instituído no Brasil no ano de 1992 pelo Ministério do Trabalho. Entretanto, o modelo convencional é fonte de polêmicas em relação à sua efetividade, sendo evidente a necessidade de alternativas que contribuam para mapas de qualidade, que auxiliem concretamente em matéria de prevenção, diminuindo acidentes e agravos à saúde do trabalhador. Neste sentido, o presente estudo aponta os riscos existentes em uma olaria no município de Caçapava do Sul/RS, apresentando uma metodologia alternativa visando propor a implementação de um mapa de riscos no empreendimento, em conjunto com os trabalhadores. Foram verificados riscos físicos como ruído, calor, vibrações e radiações não ionizantes, riscos químicos como poeira e gases no ambiente de trabalho ou resultantes de tarefas de manutenção, e, ainda, riscos ergonômicos e de acidentes, variando de acordo com o setor. Pôde-se identificar que o modelo de mapa em tela apresenta como vantagem facilitar o entendimento e a reflexão dos colaboradores sobre a existência dos diferentes riscos no exercício de suas atividades.
\end{abstract}

Palavras-Chave: Mapa de riscos; olarias; riscos ocupacionais; segurança do trabalho; trabalhadores.

\section{Resumen}

Por tratarse muchas veces de un labor en condiciones precarias y con recursos humanos con niveles de escolaridad generalmente bajos, el trabajo desarrollado en las alfarerías merece atención en lo que se refiere a la adecuada información de los trabajadores sobre los riesgos existentes en los ambientes de trabajo. En algunos casos, se observan condiciones extremadamente precarias con relación a la seguridad y salud en el trabajo, sin la debida protección de las máquinas y de los trabajadores o instalaciones eléctricas inadecuadas. El mapa de riesgos, representación gráfica de los factores capaces de acarrear perjuicios a la salud del trabajador, fue instituido en Brasil en el año 1992 por el Ministerio de Trabajo. Sin embargo, el modelo convencional es fuente de polémicas en relación a su efectividad, siendo evidente la necesidad de alternativas que contribuyan a mapas de calidad, que ayuden concretamente en materia de prevención, disminuyendo accidentes y agravios a la salud del trabajador. En este sentido, el presente estudio apunta los riesgos existentes en una alfarería en el municipio de Caçapava do Sul/RS, presentando una metodología alternativa para proponer la implementación de un mapa de riesgos en el emprendimiento, en conjunto con los trabajadores. Se han comprobado riesgos físicos como ruido, calor, vibraciones y radiaciones no ionizantes, riesgos químicos como polvo y gases en el ambiente de trabajo o resultantes de tareas de mantenimiento, y, además, riesgos ergonómicos y de accidentes, variando de acuerdo con el sector. Se pudo identificar que el modelo de mapa en

\footnotetext{
1 Especialista em Engenharia de Segurança do Trabalho; Discente do Programa de Pós-Graduação em Tecnologia Mineral - PPGTM - Universidade Federal do Pampa - UNIPAMPA; Bagé, Rio Grande do Sul, Brasil; josefagundes@unipampa.edu.br. Trabalho apresentado no III Encontro Humanístico Multidisciplinar e II Congresso Latino-Americano em Estudos Humanísticos Multidisciplinares, Jaguarão/RS, Brasil, 2017.
} 
pantalla presenta como ventaja facilitar el entendimiento y la reflexión de los colaboradores sobre la existencia de los diferentes riesgos en el ejercicio de sus actividades.

Palabras claves: Alfarerías; mapa de riesgos; riesgos ocupacionales; seguridad del trabajo; trabajadores.

\begin{abstract}
Due to the fact that they are often working in precarious conditions and with human resources with generally low levels of schooling, the work carried out in potteries deserves attention to the adequate information of the workers about the risks in the workplace. In some cases, extremely poor conditions are observed with regard to safety and health at work, without adequate protection of machines and workers or inadequate electrical installations. The risks map, a graphical representation of the factors capable of causing harm to the health of the worker, was established in Brazil in the year 1992 by the Ministry of Labor. However, the conventional model is a source of controversy regarding its effectiveness, evidencing the need for alternatives that contribute to quality maps, which specifically help in prevention, reducing accidents and injuries to workers' health. In this sense, the present study points out the existing risks in a pottery in the municipality of Caçapava do Sul/RS, presenting an alternative methodology aiming at proposing the implementation of a risks map in the enterprise, together with the workers. Have been verified physical risks such as noise, heat, vibration and non-ionizing radiations, chemical risks such as dust and gases in the work environment or resulting from maintenance tasks, as well as ergonomic and accident risks, varying according to the sector. It was possible to identify that the map model in question has the advantage of facilitate the understanding and the reflection of the employees about the existence of different risks in the exercise of their activities.
\end{abstract}

Keywords: Occupational risks; pottery; risks map; work safety; workers.

\title{
1. Introdução
}

Bentes et al. (2012) mencionam empreendimentos como olarias funcionando, em alguns casos, em condições extremamente precárias com relação à segurança e saúde no trabalho, sem a devida proteção das máquinas e dos trabalhadores, bem como com instalações elétricas inadequadas. Assim, por se tratar muitas vezes de um labor em condições precárias e com recursos humanos com níveis de escolaridade geralmente baixos, o trabalho desenvolvido nesses ambientes merece atenção no que tange à adequada informação dos colaboradores sobre os riscos existentes.

No que se refere à identificação de riscos nos ambientes de trabalho, destaca-se a ferramenta chamada mapa de riscos, instituída no Brasil através da portaria $\mathrm{n}^{\circ} 5$, de 17 de agosto de 1992, do Ministério do Trabalho (à época denominado Ministério do Trabalho e da Administração), a qual alterou a Norma Regulamentadora de Segurança e Medicina do Trabalho no 9 (NR 9) - Programa de Prevenção de Riscos Ambientais. Mattos e Freitas (1994, p.251) conceituam o mapa de riscos como uma representação gráfica do conjunto de fatores capazes de acarretar prejuízos à saúde dos trabalhadores, originados nos variados elementos do processo de trabalho, tais como materiais, equipamentos e instalações, gerados também nos espaços de trabalho, onde ocorrem as transformações, e, ainda, ocasionados pela forma de organização do trabalho, como por exemplo o arranjo físico, o ritmo de trabalho, métodos de trabalho, turnos de trabalho, postura, treinamento, etc. 
Entretanto, pode-se concordar com a observação de Mota e Frota (2013, p.496), de que o mapa de riscos é fonte de polêmicas em relação à sua efetividade nos ambientes de trabalho, sendo evidente a necessidade de alternativas e ações que contribuam para mapas de riscos de qualidade e exitosos, ou seja, que possam auxiliar concretamente em matéria de prevenção, diminuindo acidentes e agravos à saúde do trabalhador. Trata-se de uma ferramenta que depende diretamente da interpretação visual do conteúdo exposto, e, segundo esta lógica "o mapa de riscos apenas cumprirá um papel diferencial na informação e prevenção de acidentes se os trabalhadores, em sua maioria, souberem interpretá-lo corretamente." (MOTA e FROTA, 2013, p.498).

O supramencionado estudo de Mattos e Freitas (1994, p.253) questiona a portaria de 1992 como sendo objeto de muita discussão em empresas e sindicatos patronais, alegando-se dificuldades no seu cumprimento por parte dos técnicos e direções dos empreendimentos no que diz respeito a sua construção, ou seja, em relação à simbologia empregada: o uso de círculos de diferentes dimensões e cores.

Diante disso, este estudo, integrante do desenvolvimento da dissertação do Programa de Pós-Graduação em Tecnologia Mineral da Universidade Federal do Pampa, tem como objetivo inicial apresentar os riscos existentes em uma olaria no município de Caçapava do Sul/RS. Havendo outra parte, que envolve discussões em torno das medidas para eliminação, neutralização ou minimização dos riscos, o foco do presente trabalho é o entendimento do trabalhador, com a exposição dos riscos identificados nos diferentes setores da olaria e a apresentação, após, de uma metodologia alternativa de mapa de riscos para possível implementação no empreendimento, a qual visa um maior êxito no que diz respeito à prevenção no setor de transformação de minerais não metálicos.

\section{Os distintos riscos nos ambientes de trabalho}

São apontados neste trabalho os diferentes riscos aos quais os trabalhadores podem estar expostos, como os chamados riscos ambientais. "Consideram-se riscos ambientais os agentes físicos, químicos e biológicos existentes nos ambientes de trabalho que, em função de sua natureza, concentração ou intensidade e tempo de exposição, são capazes de causar danos à saúde do trabalhador.” (BRASIL, 2017).

Em relação aos agentes supramencionados, a Fundação Oswaldo Cruz - Fiocruz, instituição de ciência e tecnologia vinculada ao Ministério da Saúde, define (Fiocruz, 2017): riscos físicos, como sendo as diversas formas de energia às quais podem estar expostos os trabalhadores; riscos químicos, como o perigo ao qual o trabalhador está exposto ao 
manipular produtos que podem lhe causar danos físicos ou prejudicar sua saúde; e, riscos biológicos, como os que ocorrem por meio de microrganismos, os quais, em contato com o homem, tem a capacidade de provocar doenças.

Além dos riscos ambientais destacados, são apresentados neste estudo ainda os chamados riscos ergonômicos, bem como os riscos de acidentes existentes no empreendimento.

Para a Fiocruz, risco ergonômico é "qualquer fator que possa interferir nas características psicofisiológicas do trabalhador, causando desconforto ou afetando sua saúde." (FIOCRUZ, 2017). Podem ser elencados inúmeros agentes nesta classe, tais como esforço físico intenso, levantamento e transporte manual de peso, exigência de postura inadequada nos postos de trabalho, controle rígido de produtividade, trabalhos em turnos e noturno, jornadas de trabalho prolongadas, monotonia, repetitividade e outras situações causadoras de estresse físico e/ou psíquico.

Quanto aos riscos de acidentes, a Fiocruz os define como "todos os fatores que colocam em perigo o trabalhador ou afetam sua integridade física ou moral." (FIOCRUZ, 2017). Estes riscos possuem relação com as condições físicas do ambiente laboral, como arranjo físico inadequado, máquinas e equipamentos sem a devida proteção, ferramentas inadequadas ou defeituosas, iluminação inadequada, riscos envolvendo eletricidade, probabilidade de incêndio ou explosão, armazenamento inadequado de materiais, animais peçonhentos e outras situações de risco que podem contribuir para a ocorrência de acidentes no trabalho.

\subsection{Identificação dos riscos no empreendimento}

A seguir, são apresentados os riscos nos diferentes setores da olaria em tela. Analisaram-se as atividades desenvolvidas, identificando os agentes através de avaliação qualitativa e quantitativa.

Foi realizada coleta de dados a campo e utilizaram-se registros do Programa de Prevenção de Riscos Ambientais do empreendimento. Estes últimos, em especial, no que se refere às avaliações quantitativas dos agentes de risco físico ruído e calor.

\subsubsection{Riscos no setor de extração da matéria-prima}

No setor de extração de argila observou-se a existência de ruído, agente de risco físico, proveniente da máquina retroescavadeira. Tal agente foi avaliado quantitativamente por meio do equipamento dosímetro de ruído, resultando em um nível de $82 \mathrm{~dB}(\mathrm{~A})$. 
Também referente à máquina retroescavadeira, foi identificada a exigência de postura inadequada para sua operação, classificando-se como agente de risco ergonômico.

A Figura 1 a seguir apresenta um dos locais de extração da matéria-prima, com a retroescavadeira em operação ao fundo.

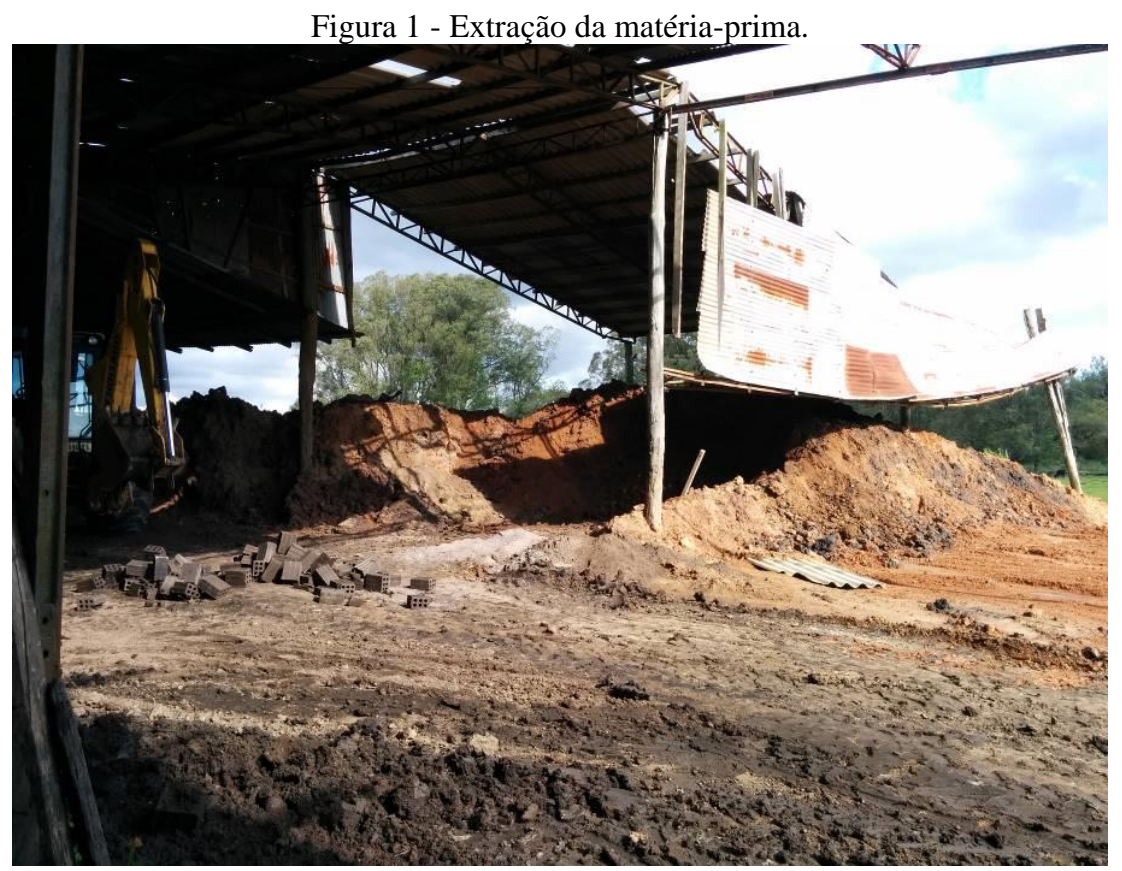

Fonte: Registro fotográfico do autor.

\subsubsection{Riscos no setor de produção de tijolos}

No setor de produção da olaria, como riscos físicos destacam-se o ruído da maromba utilizada e proveniente de postos próximos à área de extração (devido ao uso da retroescavadeira), resultando a avaliação quantitativa do equipamento dosímetro de ruído em um nível de 86,5 dB(A). Classificando-se também como agente físico, identificaram-se vibrações, quando do uso da máquina empilhadeira.

Foram verificados ainda a poeira e gases resultantes da queima da lenha e a poeira proveniente da movimentação de máquinas e materiais, sendo estes classificados como agentes de risco químico.

No que tange ao risco ergonômico, destacam-se o levantamento e transporte manual de peso no carregamento de tijolos para fornos, secadores e caminhões, bem como a postura inadequada decorrente da operação da empilhadeira.

Como riscos de acidentes podem ser elencados o risco de perda ou esmagamento de membros quando da operação de máquinas e equipamentos, risco de choques elétricos devido a instalações elétricas inadequadas, risco de atropelamento quando da máquina empilhadeira 
em movimento ou ainda o risco de queda de materiais, a depender do posto de trabalho. $\mathrm{O}$ processo de conformação das peças, etapa que apresenta risco de acidentes ao trabalhador, pode ser observado na Figura 2 a seguir.

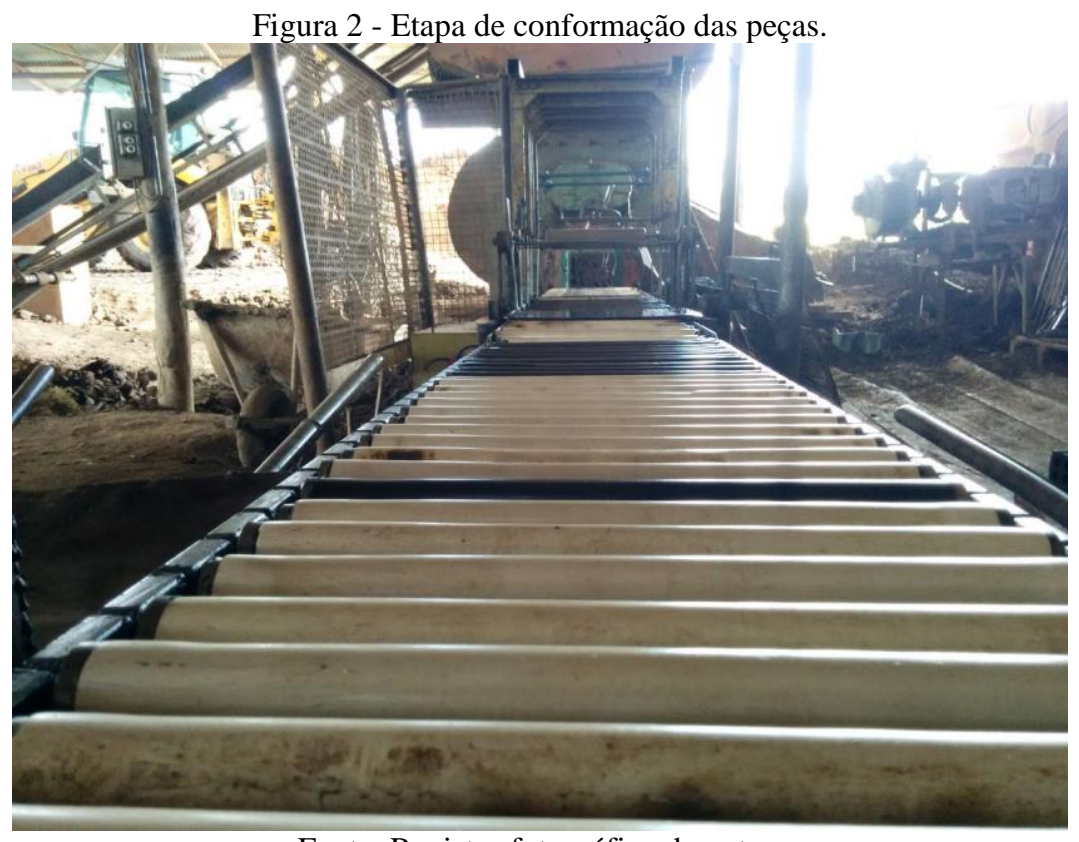

Fonte: Registro fotográfico do autor.

\subsubsection{Riscos no setor do forno/secador/queimador}

Como agente de risco físico relacionado ao forno/secador/queimador destaca-se o calor, quando do abastecimento dos fornos com lenha. Tal agente foi avaliado quantitativamente, por meio de termômetro digital específico. Com o equipamento posicionado próximo ao oleiro do setor, na altura da região de seu corpo mais atingida pelo calor, obteve-se um Índice de Bulbo Úmido Termômetro de Globo (IBUTG) de 25, $0^{\circ} \mathrm{C}$.

Também foram identificados no setor agentes de risco químico como poeira e gases, que, da mesma forma que no setor de produção, advém da queima da lenha e da movimentação de máquinas e materiais.

Como risco ergonômico, foi verificado o levantamento e transporte manual de peso no carregamento de tijolos para fornos, secadores e caminhões, bem como no carregamento de lenha para abastecimento dos fornos.

Os riscos de acidentes, neste caso, são os mesmos descritos no setor de produção (riscos de perda ou esmagamento de membros, choques elétricos, atropelamento e queda de materiais), aliados ao risco de queimaduras, principalmente em membros superiores, quando do abastecimento dos fornos com lenha.

A Figura 3, abaixo, apresenta um dos fornos da olaria. 


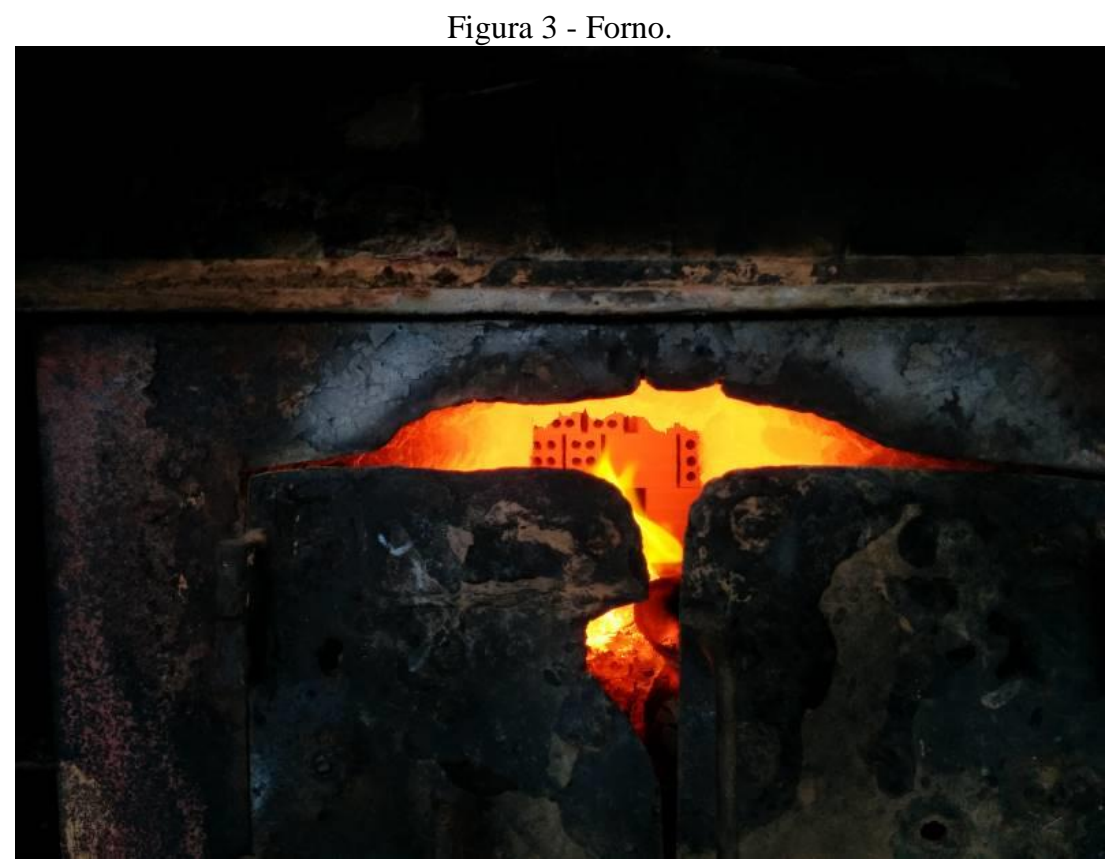

Fonte: Registro fotográfico do autor.

\subsubsection{Riscos no setor de manutenção}

Como riscos de natureza física, foram identificadas no setor de manutenção as radiações não ionizantes, provenientes das atividades envolvendo solda de materiais.

No setor, há ainda o risco decorrente do contato com hidrocarbonetos (óleos e graxas) nas atividades de manutenção de máquinas e equipamentos, bem como gases e fumos metálicos, estes últimos a depender do tipo de solda eventualmente realizada.

Como risco ergonômico, pôde-se identificar a exigência de postura inadequada, também quando do exercício das atividades de solda de materiais.

Destaca-se, ainda, o risco de perda, lesão ou esmagamento de membros na operação dos variados equipamentos utilizados no setor, classificando-se como risco de acidentes.

\subsubsection{Riscos no setor de transporte/entrega}

Nas atividades dos motoristas, profissionais que atuam realizando o transporte e entrega dos materiais produzidos na olaria, podem ser destacados o risco ergonômico, como a exigência de postura inadequada na operação do caminhão, e, ainda, o risco de acidentes, como acidentes de trânsito nos deslocamentos.

A Figura 4, abaixo, apresenta o material pronto, após a queima, o qual será transportado pelos motoristas aos clientes do empreendimento. 


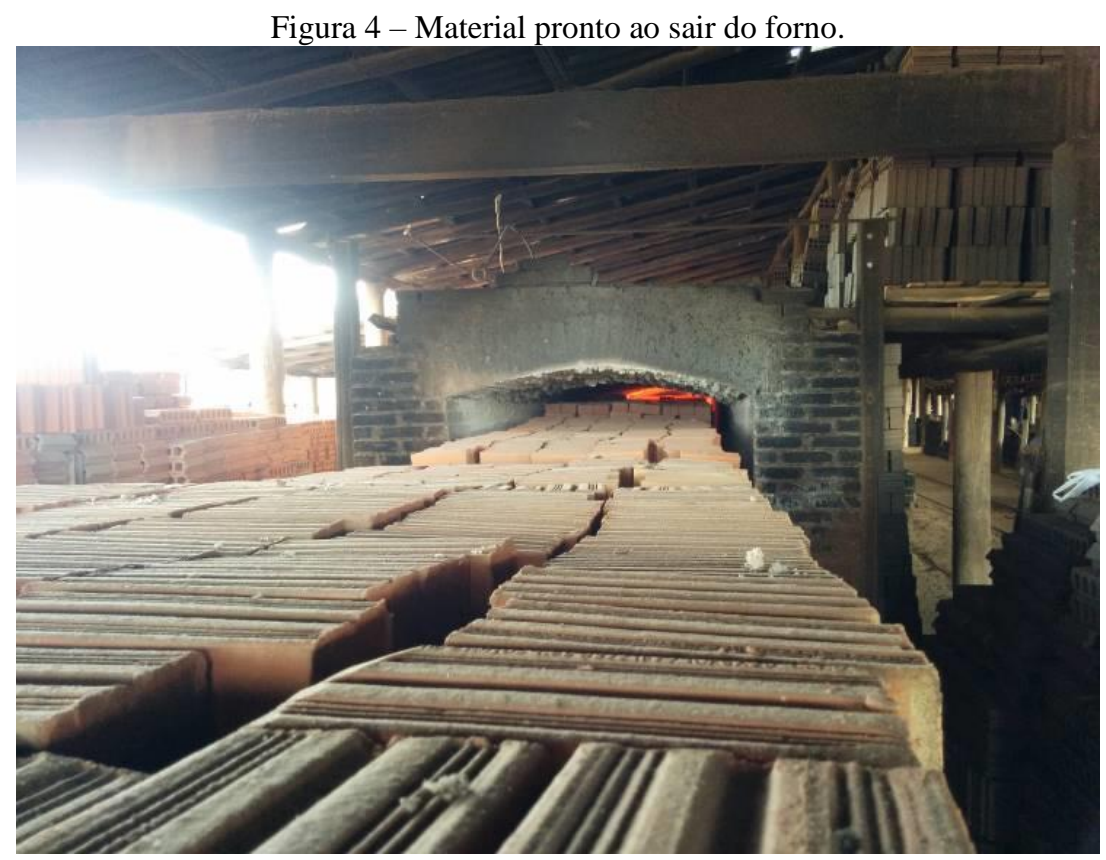

Fonte: Registro fotográfico do autor.

\subsection{Proposta alternativa de mapa de riscos}

Considerando a realidade dos riscos identificados, propõe-se a adoção pelo empreendimento de um modelo de mapa de riscos alternativo ao comumente utilizado.

Facchini et al. apud Mota e Frota (2013, p.498) propuseram uma inovação metodológica, por meio da elaboração de ícones gráficos responsáveis por representar os riscos ocupacionais, fugindo, assim, da proposta padrão de círculos. Foram desenvolvidos ícones específicos para os riscos presentes em uma indústria através da sugestão de um grupo de trabalhadores selecionados.

A Figura 5 a seguir apresenta alguns dos ícones utilizados pelos autores em seu estudo.

Figura 5 - Ícones utilizados no estudo de Facchini et al. (1997).

Riscos relacionados ao ambiente de trabalho

Fonte: Adaptado de Facchini et al. (1997, p. 500). 
Como resultado, constatou-se que os ícones propostos foram utilizados com êxito não apenas na indústria onde a metodologia foi aplicada, mas em outros estudos envolvendo trabalhadores de diferentes setores econômicos.

Facchini et al. (1997, p.499) nos dizem que o uso de círculos com cores diferentes para representar grupos de riscos segundo sua natureza acarreta em duplo prejuízo na comunicação com o trabalhador, perdendo a especificidade da exposição e a menor significação destes símbolos gráficos. Na perspectiva dos autores (Facchini et al., 1997, p.502), ao individualizar os riscos no processo de trabalho, conforme a metodologia proposta, os ícones contribuem orientando tanto atividades de prevenção e controle realizadas pelas empresas, quanto sua vigilância e fiscalização.

A Figura 6 a seguir apresenta o modelo de mapa proposto pelos autores, no caso, aplicado no setor de envase de uma indústria de laticínios.

Figura 6 - Modelo de mapa de riscos proposto por Facchini et al. (1997).

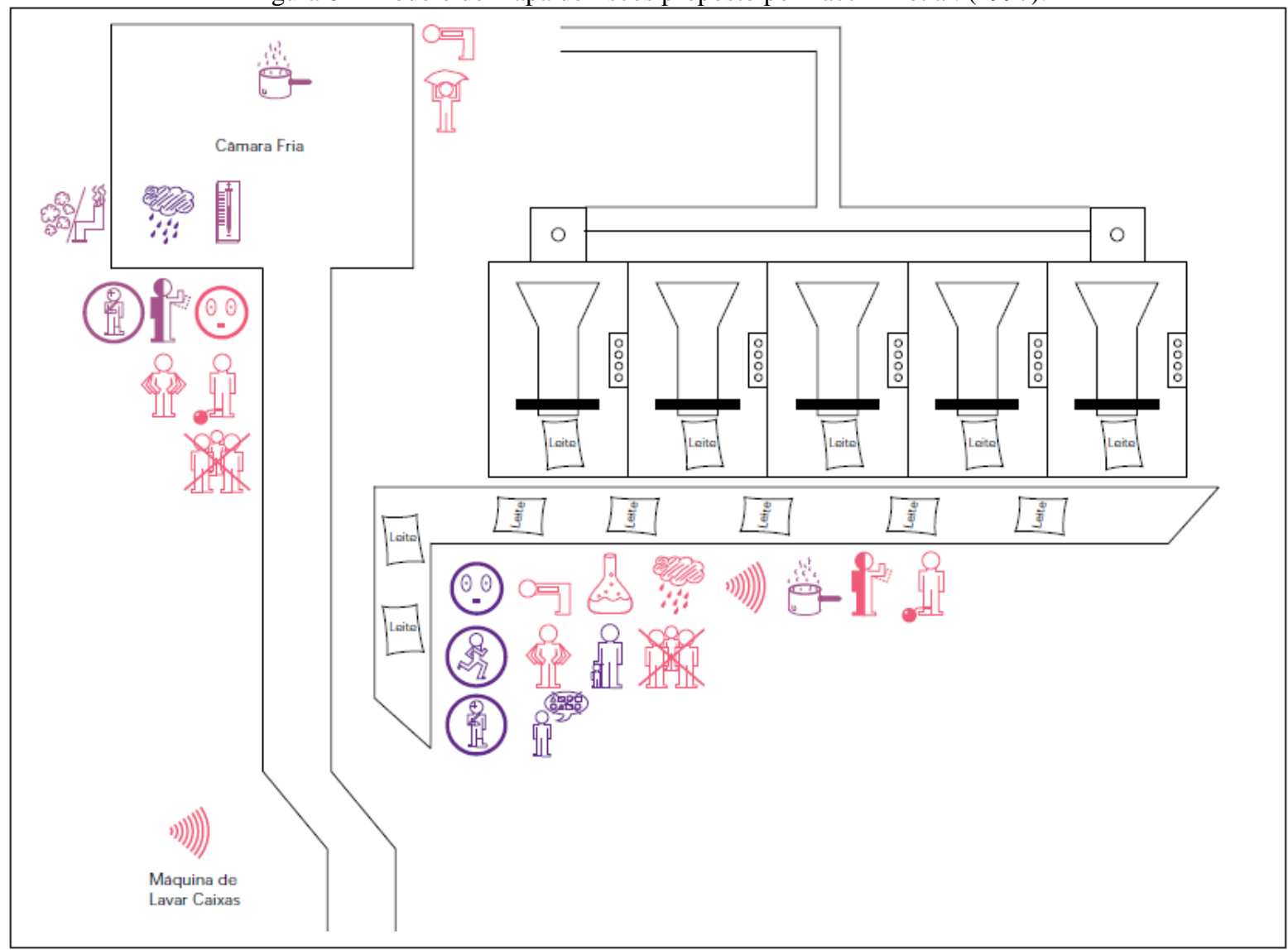

Fonte: Adaptado de Facchini et al. (1997, p. 501). 


\section{Conclusões}

Com o diagnóstico dos riscos obtido, permitiu-se identificar como principais impactos aos trabalhadores o ruído, calor, vibrações, radiações não ionizantes, poeira e gases no ambiente de trabalho ou resultantes de tarefas de manutenção, e, ainda, distintos riscos ergonômicos e de acidentes, variando de acordo com o setor do empreendimento.

Constatou-se, assim, que as características do trabalho desenvolvido na empresa provocam impactos aos recursos humanos envolvidos, sendo de suma importância a tomada de medidas a fim de eliminar, neutralizar ou minimizar os riscos identificados para a prevenção da saúde e da integridade física do homem em seu ambiente laboral.

$\mathrm{Na}$ sequencia deste estudo, pretende-se definir uma proposta de mapa de riscos baseada na metodologia alternativa apresentada, a ser construída em conjunto com os trabalhadores. Pôde-se identificar que o uso deste modelo apresenta como vantagem facilitar o entendimento e a reflexão dos colaboradores sobre a existência dos diferentes riscos no exercício de suas atividades.

\section{Referências}

BENTES, F. M.; FERREIRA, S. S.; LAMERA, D. L.; MANTOVANI, O. C.; POSSEBON, J. Cerâmica vermelha: Processo produtivo e áreas de vivência requerem melhorias. Revista Proteção (Resumo digital). Ed. 244, abr. 2012. Disponível em: http://www.protecao.com.br/edicoes/4/2012/A5ja. Acesso em: 01 dez. 2017.

BRASIL. Ministério do Trabalho. Portaria no 3.214, de 08 de junho de 1978. Normas Regulamentadoras (Internet). Brasília, DF. Disponível em: http://trabalho.gov.br/seguranca-esaude-no-trabalho/normatizacao/normas-regulamentadoras. Acesso em: 02 dez. 2017.

. Ministério do Trabalho e da Administração. Portaria DNSST n ${ }^{\circ}$ 5, de 17 de agosto de 1992. Altera a Norma Regulamentadora $n^{o} 9$ estabelecendo a obrigatoriedade de elaboração do Mapa de Riscos Ambientais. Brasília, 20 ago. 1992.

FACCHINI, L. A.; DALL'AGNOL, M. M.; FASSA, A. G.; LIMA, R. C. Ícones para mapas de riscos: uma proposta construída com os trabalhadores. Cadernos de Saúde Pública, Rio de Janeiro, v. 13, n. 3, p. 497-502, set. 1997. Disponível em: http://www.scielosp.org/scielo.php?script=sci_arttext\&pid=S0102311X1997000300025\&lng=en\&nrm=iso. Acesso em: 15 dez. 2017.

FUNDAÇÃO OSWALDO CRUZ (FIOCRUZ). Laboratório virtual. Disponível em: http://www.fiocruz.br/biosseguranca/Bis/lab_virtual/tipos_de_riscos.html. Acesso em: 11 dez. 2017.

MATTOS, U. A. O.; FREITAS, N. B. B. Mapa de risco no Brasil: as limitações da aplicabilidade de um modelo operário. Cadernos de Saúde Pública, Rio de Janeiro, v. 10, n. 2, p. 251-258, jun. 1994. Disponível em: 
http://www.scielo.br/scielo.php?script=sci_arttext\&pid=S0102-

311X1994000200012\&lng=en\&nrm=iso. Acesso em: 09 dez. 2017.

MOTA, T. T.; FROTA, O. P. A implantação do mapa de riscos no ambiente de trabalho:

revisão integrativa acerca de técnicas inovadoras. In: Revista Saúde e Pesquisa. v. 6, no 2, p. 495-501, set./dez. 2013. Universidade Católica Dom Bosco. Centro Universitário de Maringá, Maringá. 2013. 